(C) 1997 International Press

Adv. Theor. Math. Phys. 1 (1997) 237-258

\title{
D-brane Actions on Kähler Manifolds
}

\author{
Michael R. Douglas ${ }^{a}$, Akishi Kato ${ }^{b, c}$, Hirosi Ooguri ${ }^{b, d}$
}

${ }^{a}$ Department of Physics and Astronomy, Rutgers University Piscataway, NJ 08855-0849, U.S.A.

${ }^{b}$ Theoretical Physics Group, Mail Stop 50A-5101

Lawrence Berkeley National Laboratory, Berkeley, CA 94720, U.S.A.

${ }^{c}$ Department of Mathematical Sciences, University of Tokyo

Komaba 3-8-1, Meguro-ku, Tokyo 153, Japan

${ }^{d}$ Department of Physics, University of California at Berkeley 366 Le Conte Hall, Berkeley, CA 94720-7300, U.S.A.

\begin{abstract}
We consider actions for $N$ D-branes at points in a general Kähler manifold, which satisfy the axioms of D-geometry, and could be used as starting points for defining $\mathrm{M}$ (atrix)-theory in curved space.

We show that the axioms cannot be satisfied unless the metric is Ricci flat, and argue that such actions do exist when the metric is Ricci flat. This may provide an argument for Ricci flatness in M(atrix)theory.
\end{abstract}

\section{Introduction}

For many problems of D-brane physics, it is useful to know the low energy action for D-branes in curved space. Given a solution of string theory, this is defined by world-sheet computation along the lines of $[1,2]$. Such actions could also be used to define M(atrix)-theory in curved space, as discussed in [3]

For small curvatures $\alpha^{\prime} R \ll 1$, a single $\mathrm{D}$-brane in curved space is described by the supersymmetrized Nambu-Born-Infeld Lagrangian. At low 
energies, this reduces to two decoupled sectors, $U(1)$ super Yang-Mills theory and a non-linear sigma model.

A crucial feature of the D-brane is enhanced gauge symmetry when several D-branes coincide, and thus an action describing more than one D-brane in curved space must include a $U(N)$ super Yang-Mills theory as well as the non-linear sigma model, but now the two sectors are coupled.

In [4], a minimal set of axioms was proposed, which should be satisfied both by weak coupling D-brane actions in spaces of small curvature, and by $\mathrm{M}$ (atrix)-theory actions. We will review these below; they are rather uncontroversial statements, the most novel being the requirement that a string stretched between the D-branes should have mass exactly proportional to the distance between the branes. It was then shown that a $d=4, \mathcal{N}=$ $1 U(N)$ Lagrangian with one chiral superfield (parameterizing one curved complex dimension - in other words, describing a 3-brane in six dimensions) was determined (uniquely up to terms with more than two commutators) by these axioms. Dimensional reduction then produces actions for all $p$-branes with $p<3$ in six dimensions.

Here we study the analogous problem of a 3-brane in ten dimensions, with three curved complex dimensions. We will do a local analysis using normal coordinate expansions, and develop the analogous "D-normal coordinate expansion" to sixth order. We will show that the axioms cannot be satisfied unless the target space is Ricci-flat, and give strong evidence that there exists a solution when it is Ricci-flat.

Since the string theory definition works only in this case, this result might seem natural and even predestined in the context of string theory. However, since we did not use string theory to derive the action, but rather a set of axioms which make perfect sense on a general curved manifold, the result seems somewhat surprising. We will discuss its interpretation, as well as the possibility that this is a consistency condition for M(atrix)-theory compactifications, in the conclusions.

In section 2 , we review the axioms. In section 3 , we re-express these axioms as conditions on the Kähler potential and the superpotential. In section 4 , we show that these conditions imply that the target space must be Ricci flat, and that they uniquely determine the superpotential. We also find a compact expression for the conditions on the Kähler potential. In section 5 , by studying the normal coordinate expansion to the sixth order, we demonstrate that there exists a Kähler potential satisfying these conditions. It is unique at the first non-trivial (fourth) order, but at fifth order ambiguities begin to appear. Section 6 is devoted to discussion. 


\section{Axioms}

Given a $d$-dimensional Kähler manifold $\mathcal{M}$ with Kähler potential $K_{1}$, the problem will be to find a $d=4, \mathcal{N}=1 U(N)$ gauged non-linear sigma model satisfying the axioms below. The low energy action will be determined by a configuration space $\mathcal{X}_{N}$, a $d N^{2}$-dimensional Kähler manifold with potential $K_{N}$; an action of $U(N)$ by holomorphic isometries (which determines an action of $G L(N))$; and a superpotential $W$. The axioms are then

1. The classical moduli space,

$$
\left\{\mathcal{X}_{N} \mid W^{\prime}=0\right\} / / U(N)
$$

is the symmetric product $\mathcal{M}^{N} / S_{N}$.

2. The generic unbroken gauge symmetry is $U(1)^{N}$, while if two branes coincide the unbroken symmetry is $U(2) \times U(1)^{N-2}$, and so on.

3. Given two non-coincident branes at points $p_{i} \neq p_{j}$, all states charged under $U(1)_{i} \times U(1)_{j}$ have mass $m_{i j}=d\left(p_{i}, p_{j}\right)$, the distance along the shortest geodesic between the two points.

4. The action is a single trace (in terms of matrix coordinates),

$$
S=\operatorname{Tr}(\cdots)
$$

Some comments:

(i) The axioms could have been stated in a coordinate-free way. Only axiom 4 used coordinates, and it could be replaced by something like 4b: The action has no explicit $N$ dependence, and its value (on field configurations with no explicit $N$ dependence) is $O(N)$.

We will give a coordinate-free statement of the problem elsewhere, but the point is that we conjecture that the axioms imply the following statement:

Given local coordinates $z^{i}$ on $\mathcal{M}$, there exist local matrix coordinates $Z^{i}$ on $\mathcal{X}_{N}$ (we define $\bar{Z}^{i}=\left(Z^{i}\right)^{\dagger}$ ), such that the moduli space will be parameterized by diagonal matrices whose eigenvalues are coordinates on the individual copies of $\mathcal{M}$, and such that the $U(N)$ action is

$$
Z^{i} \rightarrow U^{\dagger} Z^{i} U
$$

Note that in choosing the coordinates $Z^{i}$, we must define the offdiagonal matrix elements as coordinates on $\mathcal{X}_{N}$, and (2.2) is one constraint on them. It does not completely specify them, however: we can 
still make holomorphic matrix changes of coordinate which are trivial on the moduli space:

$$
\begin{aligned}
& Z^{i} \longrightarrow W^{i}=Z^{i}+a_{j k}^{i}\left[Z^{j}, Z^{k}\right]+b_{j k l}^{i}\left[Z^{j}, Z^{k}\right] Z^{l}+\cdots, \\
& \bar{Z}^{\bar{\imath}} \longrightarrow \bar{W}^{\bar{\imath}}=\bar{Z}^{\bar{\imath}}+\bar{a}_{\bar{\jmath} \bar{k}}^{\bar{i}}\left[\bar{Z}^{\bar{k}}, \bar{Z}^{\bar{\jmath}}\right]+\bar{b}_{\bar{\jmath} k \bar{l}}^{\bar{i}} \bar{Z}^{\bar{l}}\left[\bar{Z}^{\bar{k}}, \bar{Z}^{\bar{\jmath}}\right]+\cdots
\end{aligned}
$$

where $\bar{a}_{\bar{\jmath} \bar{k}}^{\bar{i}}=\left(a_{j k}^{i}\right)^{*}$, etc. are arbitrary constants.

Thus the problem will be to find all Lagrangians of the form

$$
\mathcal{L}=\int d^{4} \theta \operatorname{Tr} K_{N}(Z, \bar{Z})+\int d^{2} \theta \operatorname{Tr} \mathcal{W}(Z)+\text { c.c. }
$$

satisfying the axioms, where we consider two Lagrangians equivalent if they are related by a field redefinition (2.3).

(ii) The potential will be the sum of that coming from the $D$-and $F$-terms, and supersymmetric vacua will satisfy the conditions

$$
\begin{aligned}
& 0=\sum_{i}\left[Z^{i}, \frac{\partial \operatorname{Tr} K_{N}}{\partial Z^{i}}\right], \\
& 0=\frac{\partial \operatorname{Tr} \mathcal{W}_{N}}{\partial Z_{i}} .
\end{aligned}
$$

We want the moduli space to be commuting matrices $\left[Z^{i}, Z^{j}\right]=0$, and in more than one dimension the $D$-flatness condition alone does not suffice to do this.

In flat space the model has the $\mathcal{N}=4, d=4$ Lagrangian, which in this notation has the superpotential $\operatorname{Tr} Z^{1}\left[Z^{2}, Z^{3}\right]$. More generally, we could take the form

$$
\mathcal{W}(Z)=\sum_{i} \epsilon_{i j k} a^{i}(Z)\left[Z^{j}, Z^{k}\right]
$$

which vanishes for any commuting matrices and thus has $\mathcal{W}^{\prime}=0$ on this subspace. For a generic function of this form, other solutions would not be expected and thus we would have the moduli space $\mathcal{M}^{N} / S_{N}$.

(iii) Clearly getting the right metric on moduli space will require us to take for $K_{N}(Z, \bar{Z})$ some version of $K(z, \bar{z})$, with a definite ordering prescription. On the moduli space, the ordering prescription will translate into a. specific dependence of the action for the off-diagonal elements on the point in moduli space.

The axioms stated can only determine the action and its second derivatives on the moduli space, since they only refer to masses of stretched 
strings, not interactions between stretched strings.

The second derivatives we will use are

$$
\left.\hat{g}_{i \bar{\jmath}}\left(z_{1}, \bar{z}_{1}, z_{2}, \bar{z}_{2}\right) \equiv \frac{\partial^{2}}{\partial Z_{12}^{i} \partial \bar{Z}_{21}^{\bar{j}}} \operatorname{Tr} K_{N}(Z, \bar{Z})\right|_{Z \in \mathcal{M}^{N} / S_{N}}
$$

and

$$
\left.\hat{\Omega}_{i j k}\left(z_{1}, z_{2}\right)\left(z_{1}-z_{2}\right)^{k} \equiv \frac{\partial^{2}}{\partial Z_{12}^{i} \partial Z_{21}^{j}} \operatorname{Tr} \mathcal{W}_{N}(Z)\right|_{Z \in \mathcal{M}^{N} / S_{N}} .
$$

(iv) We could have considered a more general gauge kinetic term,

$$
\operatorname{Re} \int d^{2} \theta \operatorname{Tr} f(Z) W^{2}+f_{i j}(Z)\left[W, Z^{i}\right]\left[W, Z^{j}\right] .
$$

Taking non-constant $f(Z)$ would lead to a space-dependent gauge coupling, as would come from a non-constant dilaton background. The mass conditions in this case involve the dilaton as well as the metric (e.g. see [5]), and we will not consider this case here. Non-constant $f_{i j}(Z)$, as we will see below, turns out to be incompatible with the mass conditions.

\section{Mass Conditions on Kähler Potential and Su- perpotential}

In this section, we summarize conditions on the Kähler potential and superpotential imposed by the axioms. In particular, we express the mass condition (the axiom 3 ) as conditions on $\hat{g}\left(z_{1}, z_{2}\right)$ and $\hat{\Omega}_{i j k}\left(z_{1}, z_{2}\right)$ defined in (2.7 and (2.8).

Our considerations will be in a neighborhood of a point $p$ in the target space, and we will use coordinates $z$ in which this is at $z=0$.

\subsection{Normal Coordinates and the Squared Distance}

On any Kähler manifold $\mathcal{M}$, one can always find a holomorphic local coordinate system (normal coordinates) such that the metric has an expansion around $z=0$ as

$$
g_{i \bar{\jmath}}(z, \bar{z})=\delta_{i \bar{\jmath}}+\sum_{p=1}^{\infty} \sum_{q=1}^{\infty} g_{i \bar{\jmath} ; k_{1} \ldots k_{p} ; \bar{l}_{1} \ldots \bar{l}_{q}}^{(p, q)} z^{k_{1}} \cdots z^{k_{p}} \bar{z}^{\bar{l}_{1}} \cdots \bar{z}^{\bar{l}_{q}}
$$

By using Kähler potential transformations $K_{1} \rightarrow K_{1}+\operatorname{Re} F(z)$, we can assume the Kähler potential on $\mathcal{M}$ has an expansion

$$
K_{1}(z, \bar{z})=|z|^{2}+\sum_{p=2}^{\infty} \sum_{q=2}^{\infty} K_{1 ; k_{1} \ldots k_{p} ; \bar{l}_{1} \ldots \bar{l}_{q}}^{(p, q)} z^{k_{1}} \cdots z^{k_{p}} \bar{z}^{\bar{l}_{1}} \cdots \bar{z}^{\bar{l}_{q}} .
$$


More explicitly,

$$
K_{1}(z, \bar{z})=z^{i} \bar{z}^{i}-\frac{1}{4} R_{i \jmath k l} z^{i} \bar{z}^{\bar{\jmath}} z^{k} \bar{z}^{\bar{l}}+\ldots
$$

where $R_{i j k l}$ is the Riemann curvature at $z=0$. All symmetries of the Riemann tensor, $R_{i \bar{\jmath} k \bar{l}}=R_{k \bar{\jmath} i \bar{l}}$ and $R_{i \bar{\jmath} k \bar{l}}=R_{i \bar{l} \bar{k} \bar{\jmath}}$, are manifest in this expression.

We also need an expression for the geodesic distance. Let $d^{2}(x, z)$ be the squared geodesic distance from $x$ to $z$. It obeys the differential equation

$$
g^{i \bar{\jmath}}(x) \frac{\partial d^{2}(x, z)}{\partial x^{i}} \frac{\partial d^{2}(x, z)}{\partial \bar{x}^{\bar{j}}}=d^{2}(x, z)
$$

which follows from the Einstein relation $4 g^{i \bar{j}} p_{i} p_{\bar{\jmath}}=m^{2}$, the momentum $p_{i}=$ $m \partial S(x, y) / \partial x^{i}$ and the fact that the point particle action is proportional to the geodesic distance, $S(x, y)=m d(x, y)$.

This combined with the initial condition

$$
\left.\frac{\partial^{2} d^{2}(x, z)}{\partial x^{i} \partial \bar{x}^{\bar{\jmath}}}\right|_{z=x}=g_{i \bar{\jmath}}(x),
$$

determines the geodesic distance uniquely [6]. For example, its expansion to $O\left(z^{4}\right)$ is

$$
\begin{aligned}
d^{2}(x, z)=|x-z|^{2}-\frac{1}{4} R_{i \bar{\jmath} k l}(x-z)^{i}(\bar{x}-\bar{z})^{\bar{\jmath}}(x+z)^{k}(\bar{x}+\bar{z})^{\bar{l}} \\
\quad-\frac{1}{12} R_{i \bar{\jmath} k l}(x-z)^{i}(\bar{x}-\bar{z})^{\bar{\jmath}}(x-z)^{k}(\bar{x}-\bar{z})^{\bar{l}}+\ldots
\end{aligned}
$$

In section 6 , the expansion to the sixth order is given.

According to axiom 4, the Kähler potential $K_{N}$ for the $N$ D-branes is expressed as a trace of a power series in $Z$ and $\bar{Z}$. Again, by a holomorphic coordinate change $Z \rightarrow f(Z)$, we can go to normal coordinates on the full configuration space $\mathcal{X}_{N}$, in which $K_{N}$ takes the form

$$
\operatorname{Tr} K_{N}(Z, \bar{Z})=\operatorname{Tr} Z^{i} \bar{Z}^{i}+\sum_{p, q=2}^{\infty} K_{N}^{(p, q)} \operatorname{Tr} Z \cdots \bar{Z} \cdots Z \cdots \bar{Z} \cdots
$$

Here $Z \cdots \bar{Z} \cdots Z \cdots \bar{Z} \cdots$ is some sequence of $p Z$ 's and $q \bar{Z}$ 's. This eliminates the ambiguity expressed in equation (2.3).

As cliscussed in [4], reproducing the metric for each of the $N$ branes requires

$$
\left.\operatorname{Tr} K_{N}(Z, \bar{Z})\right|_{Z \in \mathcal{M}^{N} / S_{N}}=\sum_{a=1}^{N} K_{1}\left(z_{a}, \bar{z}_{a}\right),
$$

where $z_{1}^{i}, \ldots, z_{N}^{i}$ are the eigenvalues of $Z^{i}$. Thus $K_{N}$ must have the same expansion in powers of $Z$ and $\bar{Z}$ as $K_{1}$, but the precise ordering remains to be fixed. 
A natural guess for the full Kähler form would be

$$
\begin{aligned}
\operatorname{Tr} K_{N}(Z, \bar{Z}) & =\operatorname{STr} K_{1}(Z, \bar{Z}) \\
& =\operatorname{Tr} Z^{i} \bar{Z}^{\bar{\imath}}-\frac{1}{4} R_{i \bar{\jmath} k l} \operatorname{STr} Z^{i} \bar{Z}^{\bar{j}} Z^{k} \bar{Z}^{\bar{l}}+\cdots,
\end{aligned}
$$

where $\mathrm{S} \operatorname{Tr}$ is the symmetrized trace: $\mathrm{S} \operatorname{Tr} A_{1} \cdots A_{n}=\frac{1}{n !} \sum_{\sigma \in S_{N}} \operatorname{Tr} A_{\sigma(1)} \cdots$ $A_{\sigma(n)}$. However, there could be additional terms which vanish on the moduli space, and we will need to constrain them by using the mass condition (axiom 3 ). As it will turn out, (3.9) must be corrected (at sixth order!).

\subsection{The Mass Condition for Gauge Bosons}

The simplest thing to check is that the gauge boson masses are correctly reproduced, because these do not depend on the superpotential. This requires

$$
d^{2}\left(z_{1}, z_{2}\right)=\left.\frac{1}{2} \frac{d^{2}}{d t d \bar{t}} \operatorname{Tr} K_{N}(Z+t[X, Z], \bar{Z}+\bar{t}[X, \bar{Z}])\right|_{Z \in \mathcal{M}^{N} / S_{N}, t=0},
$$

where $X=E_{12}+E_{21}$ is a broken generator. More explicitly,

$$
\hat{g}_{i \bar{\jmath}}\left(z_{1}, \bar{z}_{1}, z_{2}, \bar{z}_{2}\right)\left(z_{1}-z_{2}\right)^{i}\left(\bar{z}_{1}-\bar{z}_{2}\right)^{\bar{\jmath}}=d^{2}\left(z_{1}, z_{2}\right) .
$$

\subsection{Non-Constant Gauge Kinetic Term}

We now consider the case of non-constant $f_{i j}(z)$ in (2.9). This would modify the mass condition to

$$
\hat{g}_{i \bar{\jmath}}\left(z_{1}, \bar{z}_{1}, z_{2}, \bar{z}_{2}\right)\left(z_{1}-z_{2}\right)^{i}\left(\bar{z}_{1}-\bar{z}_{2}\right)^{\bar{\jmath}}=d^{2}\left(z_{1}, z_{2}\right) \operatorname{Re} f\left(z_{1}, z_{2}\right),
$$

where $f\left(z_{1}, z_{2}\right)$ is holomorphic.

Suppose $f\left(z_{1}, z_{2}\right) \sim 1+z^{k}$ is non-constant; then the left hand side will include a term $O\left(z^{k+1} \bar{z}\right)+$ c.c.. From (3.7), we see that in normal coordinates $\hat{g}_{i \bar{\jmath}} \sim \delta_{i \bar{\jmath}}+O(z \bar{z})$, leading to the right hand side $|z|^{2}+O\left(z^{2} \bar{z}^{2}\right)$ which cannot include such a term.

Thus the mass condition requires trivial gauge kinetic term, and we restrict attention to this case.

\subsection{The Mass Condition for Scalars}

The masses of the chiral superfields are determined by the kinetic term $\hat{g}_{i \bar{j}}$ and the second derivatives of the potential, and thus their mass condition is

$$
\delta_{i} \bar{\delta}_{\bar{\jmath}} V=\delta_{i} \bar{\delta}_{\bar{\jmath}} V_{D}+\delta_{i} \bar{\delta}_{\bar{\jmath}} V_{F} \equiv d^{2} \hat{g}_{i \bar{\jmath}},
$$

where $\delta_{i}=\partial / \partial Z_{12}^{i}$ and $\bar{\delta}_{\bar{\jmath}}=\partial / \partial \bar{Z}_{21}^{\bar{j}}$. 
The $D$-term contribution is

$$
V_{D}=-\frac{1}{2} \sum_{i, \bar{\jmath}} \operatorname{Tr}\left[Z^{i}, \frac{\partial K}{\partial Z^{i}}\right]\left[\bar{Z}^{\bar{j}}, \frac{\partial K}{\partial \bar{Z}^{\bar{j}}}\right] .
$$

and its second derivative on the moduli space was given in [4], while the $F$-term contribution is

$$
V_{F}=\left(\frac{\partial^{2} K}{\partial Z_{a b}^{i} \partial \bar{Z}_{b a}^{j}}\right)^{-1} \frac{\partial W}{\partial Z_{a b}^{i}} \frac{\partial \bar{W}}{\partial \bar{Z}_{b a}^{j}}
$$

with second derivative on the moduli space

$$
\begin{aligned}
\frac{\partial^{2} V_{F}}{\partial Z_{12}^{i} \partial \bar{Z}_{21}^{\bar{j}}} & =\left(\frac{\partial^{2} K}{\partial Z_{12}^{k} \partial \bar{Z}_{21}^{\bar{m}}}\right)^{-1} \frac{\partial^{2} W}{\partial Z_{12}^{i} \partial Z_{21}^{k}} \frac{\partial^{2} \bar{W}}{\partial \bar{Z}_{21}^{\bar{j}} \partial Z_{12}^{\bar{m}}} \\
& =\hat{g}^{k \bar{m}} \hat{\Omega}_{i k l}\left(z_{1}^{l}-z_{2}^{l}\right) \hat{\Omega}_{\bar{j} \bar{m} \bar{n}}\left(\bar{z}_{1}^{\bar{n}}-\bar{z}_{2}^{\bar{n}}\right) .
\end{aligned}
$$

More explicitly, with the gauge boson mass (3.11), the mass condition (3.13) reads

$$
\begin{aligned}
& \hat{g}_{i \bar{n}(}\left(\bar{z}_{1}^{\bar{n}}-\bar{z}_{2}^{\bar{n}}\right) \hat{g}_{m \bar{\jmath}}\left(z_{1}^{m}-z_{2}^{m}\right)+\hat{g}^{k \bar{l}} \hat{\Omega}_{i k m}\left(z_{1}^{m}-z_{2}^{m}\right) \hat{\Omega}_{\bar{\jmath} \bar{l}}\left(\bar{z}_{1}^{\bar{n}}-\bar{z}_{2}^{\bar{n}}\right) \\
& \quad \equiv \hat{g}_{i \bar{\jmath}} \hat{g}_{m \bar{n}}\left(z_{1}^{m}-z_{2}^{m}\right)\left(\bar{z}_{1}^{\bar{n}}-\bar{z}_{2}^{\bar{n}}\right)
\end{aligned}
$$

\section{More on the Mass Conditions}

In this section, we study the mass conditions (3.11) and (3.17) found in the previous section. We show that these require that the target space be Ricci-flat and also determine the superpotential completely.

\subsection{Ricci Flatness}

In this subsection, we will show that the mass condition (3.17) implies $R_{i \bar{\jmath}}=$ 0 .

Note that the off-diagonal metric $\hat{g}$ and diagonal metric $g$ are related as

$$
\lim _{z_{1} \rightarrow z_{2}} \hat{g}_{i \bar{\jmath}}\left(z_{1}, \bar{z}_{1}, z_{2}, \bar{z}_{2}\right)=g_{i \bar{\jmath}}\left(z_{2}, \bar{z}_{2}\right)
$$

which follows from (3.5) and (3.11). For later convenience, set

$$
\Omega_{i j k}\left(z_{2}\right)=\lim _{z_{1} \rightarrow z_{2}} \frac{\partial}{\partial z_{1}^{k}}\left\{\hat{\Omega}_{i j m}\left(z_{1}, z_{2}\right)\left(z_{1}-z_{2}\right)^{m}\right\} .
$$


Taking $\lim _{z_{1} \rightarrow z_{2}} \frac{\partial^{2}}{\partial z_{1}^{m} \partial \bar{z}_{2}^{\tilde{r}}}$ of $(3.17)$, we get

$$
g_{i \bar{n}} g_{m \bar{\jmath}}+g^{k \bar{l}} \Omega_{i k m} \Omega_{\bar{\jmath} \bar{l} \bar{n}}=g_{i \bar{\jmath}} g_{m \bar{n}} .
$$

As was explained in the introduction, we assume a superpotential of the form

$$
\mathcal{W}_{N}(Z)=c \operatorname{Tr} Z^{1}\left[Z^{2}, Z^{3}\right]+\cdots
$$

where $\cdots$ stands for higher order terms with at least one commutator. Then, $\Omega_{i j k}(z)$ can be expanded as

$$
\Omega_{i j k}(z)=c \epsilon_{i j k}+\sum_{p \geq 1} \Omega_{i j k ; m_{1} \ldots m_{p}}^{(p)} z^{m_{1}} \cdots z^{m_{p}} .
$$

Let us plug the expansions (3.1) and (4.4) into (4.2). On the right hand side, there are no purely holomorphic terms in the expansion. On the left hand side, however, such terms would appear with coefficients $\delta^{k \bar{l}} \Omega_{i k m ; m_{1} \cdots m_{p}}^{(p)} \epsilon_{\bar{j} \bar{l} \bar{n}}$. This implies $\Omega_{i k m ; m_{1} \cdots m_{p}}^{(p)}=0$ for all $p \geq 1$, i.e. $\Omega_{i j k}(z)$ actually has no $z$ dependence in our coordinate system;

$$
\Omega_{i j k}(z)=c \epsilon_{i j k} .
$$

Then, (4.2) reduces to

$$
g_{i \bar{n}} g_{m \bar{\jmath}}+|c|^{2} g^{k \bar{l}} \epsilon_{i k m} \epsilon_{\bar{\jmath} \bar{l} \bar{n}}=g_{i \bar{j}} g_{m \bar{n}}
$$

which is equivalent to $\operatorname{det} g_{m \bar{n}}(z, \bar{z})=|c|^{2}$. The normalization (3.1) fixes $|c|=1$. Thus the final result is

$$
\operatorname{det} g_{m \bar{n}}(z, \bar{z}) \equiv 1 .
$$

Ricci flatness of $\mathcal{M}$ is a corollary of this:

$$
R_{i \bar{\jmath}} \equiv \frac{\partial^{2}}{\partial z^{i} \partial \bar{z}^{\bar{\jmath}}} \log \operatorname{det} g_{m \bar{n}}(z, \bar{z})=0 .
$$

\subsection{Superpotential $\mathcal{W}_{N}(Z)$}

In this subsection we will show that the mass condition (3.17) determines the superpotential $\operatorname{Tr} \mathcal{W}_{N}(Z)$ to be

$$
\operatorname{Tr} \mathcal{W}_{N}(Z)=\operatorname{Tr} Z^{1}\left[Z^{2}, Z^{3}\right],
$$

to all orders in diagonal coordinates but up to the second order in commutators. 
The basic strategy is quite similar to that used in the previous subsection. Here, instead of taking the limit $z_{1} \rightarrow z_{2}$, we will expand various quantities as a power series in $z_{1}-z_{2}$ and $\bar{z}_{1}-\bar{z}_{2}$ :

$$
\begin{aligned}
A\left(z_{1}, \bar{z}_{1}, z_{2}, \bar{z}_{2}\right)=\sum_{p, q=0}^{\infty} & A_{i_{1} \cdots i_{p} ; \bar{j}_{1} \cdots \bar{j}_{q}}^{\left(p, z_{1}\right.}\left(\frac{z_{1}+z_{2}}{2}, \frac{\bar{z}_{1}+\bar{z}_{2}}{2}\right) \\
& \times\left(z_{1}-z_{2}\right)^{i_{1}} \cdots\left(z_{1}-z_{2}\right)^{i_{p}}\left(\bar{z}_{1}-z_{2}\right)^{\bar{j}_{1}} \cdots\left(\bar{z}_{1}-z_{2}\right)^{\bar{J}_{q}} .
\end{aligned}
$$

The second derivative of the superpotential is expanded as

$$
\begin{aligned}
\hat{\Omega}_{i j k}\left(z_{1}-z_{2}\right)^{k} & =\left.\frac{\partial^{2}}{\partial Z_{12}^{i} \partial Z_{21}^{j}} \operatorname{Tr} \mathcal{W}_{N}(Z)\right|_{Z \in \mathcal{M}^{N} / S_{N}} \\
& =\sum_{p=1}^{\infty} \hat{\Omega}_{i j ; k_{1} \ldots k_{p}}^{(p)}\left(\frac{z_{1}+z_{2}}{2}\right)\left(z_{1}-z_{2}\right)^{k_{1}} \cdots\left(z_{1}-z_{2}\right)^{k_{p}} .
\end{aligned}
$$

From the superpotential (4.3), we have

$$
\hat{\Omega}_{i j ; k}^{(1)}\left(\frac{z_{1}+z_{2}}{2}\right)=c \epsilon_{i j k}+O\left(\frac{z_{1}+z_{2}}{2}\right) .
$$

We saw in the previous subsection that $|c|=1$ and by choice of coordinates we can take $c=1$. Then, (4.5) implies

$$
\Omega_{i j k}\left(z_{2}\right)=\lim _{z_{1} \rightarrow z_{2}} \frac{\partial}{\partial z_{1}^{k}}\left\{\hat{\Omega}_{i j m}\left(z_{1}, z_{2}\right)\left(z_{1}-z_{2}\right)^{m}\right\}=\Omega_{i j ; k}^{(1)}\left(z_{2}\right)=\epsilon_{i j k} .
$$

$\delta_{i} \delta_{j} \operatorname{Tr} \mathcal{W}_{N}$ now reads

$$
\hat{\Omega}_{i j k}\left(z_{1}-z_{2}\right)^{k}=\epsilon_{i j k}+\sum_{p=2}^{\infty} \hat{\Omega}_{i j ; k_{1} \ldots k_{p}}^{(p)}\left(\frac{z_{1}+z_{2}}{2}\right)\left(z_{1}-z_{2}\right)^{k_{1}} \cdots\left(z_{1}-z_{2}\right)^{k_{p}} .
$$

On the other hand, the D-Kähler potential (3.7) gives the following expansion of $\hat{g}$

$$
\begin{aligned}
\hat{g}_{i \bar{\jmath}}\left(z_{1}, \bar{z}_{1}, z_{2}, \bar{z}_{2}\right)=\delta_{i \bar{\jmath}} & \sum_{p=1}^{\infty} \sum_{q=1}^{\infty} \hat{g}_{i \bar{\jmath} ; k_{1} \ldots k_{p} ; \bar{l}_{1} \ldots \bar{l}_{q}}^{(p, q)}\left(\frac{z_{1}+z_{2}}{2}, \frac{\bar{z}_{1}+\bar{z}_{2}}{2}\right) \\
& \times\left(z_{1}-z_{2}\right)^{k_{1}} \cdots\left(z_{1}-z_{2}\right)^{k_{p}}\left(\bar{z}_{1}-z_{2}\right)^{\bar{l}_{1}} \cdots\left(\bar{z}_{1}-z_{2}\right)^{\bar{l}_{q}} .
\end{aligned}
$$

Let us consider a similar expansion of the mass degeneracy condition (3.17). On the right hand side, there are no terms with bi-degree $(p=1, q \geq$ $2)$. On the left hand side, however, such terms would appear with coefficients $\delta^{k l} \epsilon_{\bar{J} \bar{l}} \Omega_{i k ; m_{1} \cdots m_{p}}^{(p)}$. Thus, $\hat{\Omega}^{(p)}$ must vanish for all $p \geq 2$. This implies

$$
\hat{\Omega}_{i j k}\left(z_{1}, z_{2}\right)\left(z_{1}-z_{2}\right)^{k}=\epsilon_{i j k}\left(z_{1}-z_{2}\right)^{k},
$$

to all orders in $z_{1}$ and $z_{2}$, and the stated result. 


\subsection{Condition on $\operatorname{det} \hat{g}$}

Given the $F$ term contribution (4.13), we can reduce the mass condition (3.17) to a much simpler form:

$$
\begin{aligned}
& \hat{g}_{i \bar{n}} \hat{g}_{m \bar{\jmath}}\left(z_{1}^{m}-z_{2}^{m}\right)\left(\bar{z}_{1}^{\bar{n}}-\bar{z}_{2}^{\bar{n}}\right)+\hat{g}^{k \bar{l}} \epsilon_{i k m} \epsilon_{\bar{\jmath} \bar{n}}\left(z_{1}^{m}-z_{2}^{m}\right)\left(\bar{z}_{1}^{\bar{n}}-\bar{z}_{2}^{\bar{n}}\right) \\
& \quad \equiv \hat{g}_{i \bar{\jmath}} \hat{g}_{m \bar{n}}\left(z_{1}^{m}-z_{2}^{m}\right)\left(\bar{z}_{1}^{\bar{n}}-\bar{z}_{2}^{\bar{n}}\right)
\end{aligned}
$$

which can be rewritten as

$$
(1-\operatorname{det} \hat{g}) \times \hat{g}^{k \bar{l}} \epsilon_{i k m} \epsilon_{\bar{\jmath} \bar{l} \bar{n}}\left(z_{1}^{m}-z_{2}^{m}\right)\left(\bar{z}_{1}^{\bar{n}}-\bar{z}_{2}^{\bar{n}}\right)=0 .
$$

This holds if and only if

$$
\operatorname{det} \hat{g}\left(z_{1}, \bar{z}_{1}, z_{2}, \bar{z}_{2}\right) \equiv 1
$$

The condition (4.16) bears a striking similarity to the previous result $\operatorname{det} g(z, \bar{z}) \equiv 1$. This is consistent since $g$ and $\hat{g}$ are related via (4.1).

\section{Explicit Form of D-Kähler Potential}

We have seen that the axioms of D-geometry and especially the mass condition (3.17) puts stringent constraints on our problem. In normal coordinates, the superpotential $\mathcal{W}_{N}(Z)$ is uniquely fixed as (4.8) and the nontrivial information of D-geometry is encoded in the Kähler potential $K_{N}(Z, \bar{Z})$.

Let us summarize the properties $K_{N}(Z, \bar{Z})$ should have:

1. It must reproduce the Ricci flat metric for each of the $N$ branes.

$$
\left.\operatorname{Tr} K_{N}(Z, \bar{Z})\right|_{Z \in \mathcal{M}^{N} / S_{N}}=\sum_{a=1}^{N} K_{1}\left(z_{a}, \bar{z}_{a}\right)
$$

where $z_{1}^{i}, \ldots, z_{N}^{i}$ are the eigenvalues of $Z^{i}$.

2. The gauge boson mass condition: Its second derivative must reproduce the geometric distance.

$$
\hat{g}_{i \bar{\jmath}}\left(z_{1}, \bar{z}_{1}, z_{2}, \bar{z}_{2}\right)\left(z_{1}-z_{2}\right)^{i}\left(\bar{z}_{1}-\bar{z}_{2}\right)^{\bar{\jmath}}=d^{2}\left(z_{1}, z_{2}\right)
$$

3.

$$
\operatorname{det} \hat{g}_{i \bar{\jmath}}\left(z_{1}, \bar{z}_{1}, z_{2}, \bar{z}_{2}\right) \equiv 1
$$




\section{1 $K_{N}$ to the Fourth Order}

We now show that, at the first non-trivial order (fourth order) in the normal coordinate expansion, there exists a unique $K_{N}$ satisfying these conditions:

$$
K_{N}(Z, \bar{Z})=\operatorname{Tr} Z^{i} \bar{Z}^{\bar{\imath}}-\frac{1}{4} R_{i \bar{\jmath} k l} \mathrm{~S} \operatorname{Tr} Z^{i} Z^{k} \bar{Z}^{\bar{\jmath}} \bar{Z}^{\bar{l}} .
$$

The most general form of $K_{N}$ to fourth order reads

$$
\begin{aligned}
\operatorname{Tr} K_{N}(Z, \bar{Z})=\operatorname{Tr} Z \bar{Z} & -\frac{1}{4} R_{i \bar{\jmath} k l} \operatorname{STr} Z^{i} Z^{k} \bar{Z}^{j} \bar{Z}^{\bar{l}} \\
& +\frac{1}{4} A_{(i j)[\bar{k} l]} \operatorname{Tr}\left\{Z^{i}, Z^{j}\right\}\left[\bar{Z}^{\bar{k}}, \bar{Z}^{\bar{l}}\right] \\
& +\frac{1}{4} A_{[i j](\bar{k} \bar{l})}^{*} \operatorname{Tr}\left[Z^{i}, Z^{j}\right]\left\{\bar{Z}^{\bar{k}}, \bar{Z}^{\bar{l}}\right\} \\
& +\frac{1}{4} B_{[i j][\bar{k} l]} \operatorname{Tr}\left[Z^{i}, Z^{j}\right]\left[\bar{Z}^{\bar{k}}, \bar{Z}^{\bar{l}}\right] \\
& +\frac{1}{2} C_{(i k)(\bar{\jmath} \bar{l})} \operatorname{Tr}\left[Z^{i}, \bar{Z}^{\bar{j}}\right]\left[Z^{k}, \bar{Z}^{\bar{l}}\right] \\
& +D_{[i k][\bar{\jmath}]} \operatorname{Tr} Z^{i} \bar{Z}^{\bar{\jmath}} Z^{k} \bar{Z}^{\bar{l}} .
\end{aligned}
$$

In general, the terms $B$ and $C$ together are redundant, by using the Jacobi identity. For example, $\operatorname{Tr}[x, \bar{x}][y, \bar{y}]-\operatorname{Tr}[x, \bar{y}][y, \bar{x}]=\operatorname{Tr}[x, y][\bar{x}, \bar{y}]$. So, we require that $C$ be symmetric under such interchanges. Similarly, the symmetry condition on $D$ makes it independent of $C$ and $R$.

To check that this includes all fourth order terms, we count $d^{4}$ terms $\operatorname{Tr} Z^{i} Z^{j} \bar{Z}^{k} \bar{Z}^{l}$ and $d^{2}\left(d^{2}+1\right) / 2$ of the form $\operatorname{Tr} Z^{i} \bar{Z}^{\bar{j}} Z^{k} \bar{Z}^{\bar{l}}$, while the expansion above has $(d(d+1) / 2)^{2}+2(d(d-1) / 2)^{2}+2(d(d-1) / 2)(d(d+1) / 2)+(d(d+$ 1) $/ 2)^{2}$ terms, which agrees.

To check the conditions at fourth order we use (3.6) for $d^{2}$, and compute (2.7 using (5.5) to find

$$
\begin{aligned}
\hat{g}_{i \bar{\jmath}}\left(z_{1}, \bar{z}_{1}, z_{2}, \bar{z}_{2}\right)=\delta_{i \bar{\jmath}} & -\frac{1}{4} R_{i \overline{j k} \bar{l}}\left(z_{1}+z_{2}\right)^{k}\left(\bar{z}_{1}+\bar{z}_{2}\right)^{\bar{l}} \\
& -\frac{1}{12} R_{i \bar{\jmath} k \bar{l}}\left(z_{1}-z_{2}\right)^{k}\left(\bar{z}_{1}-\bar{z}_{2}\right)^{\bar{l}} \\
& +\left(A_{i k \bar{\jmath} \bar{l}}+D_{i k \bar{l} \bar{l}}\left(z_{1}+z_{2}\right)^{k}\left(\bar{z}_{1}-\bar{z}_{2}\right)^{\bar{l}}\right. \\
& +\left(A_{i k \bar{\jmath} \bar{l}}^{*}+D_{i k \bar{l}}\right)\left(z_{1}-z_{2}\right)^{k}\left(\bar{z}_{1}+\bar{z}_{2}\right)^{\bar{l}} \\
& +B_{i k \bar{\jmath} \bar{l}}\left(z_{1}-z_{2}\right)^{k}\left(\bar{z}_{1}-\bar{z}_{2}\right)^{\bar{l}} \\
& -C_{i k \bar{l} \bar{\jmath}}\left(z_{1}-z_{2}\right)^{k}\left(\bar{z}_{1}-\bar{z}_{2}\right)^{\bar{l}} .
\end{aligned}
$$

We find that, taking into account the symmetries of the tensors, we need $A=C=D=0$ but $B$ is undetermined. 
Now let us use condition 3. Using our computation (5.6) of $\hat{g}$, we have (to second order)

$\operatorname{det} \hat{g}_{i \bar{\jmath}}=1-\frac{1}{4} R_{k \bar{l}}\left(z_{1}+z_{2}\right)^{k}\left(\bar{z}_{1}+\bar{z}_{2}\right)^{\bar{l}}-\left(\frac{1}{12} R_{k \bar{l}}-B_{k \bar{l}}\right)\left(z_{1}-z_{2}\right)^{k}\left(\bar{z}_{1}-\bar{z}_{2}\right)^{\bar{l}}$,

where $R_{k \bar{l}}=\delta^{i \bar{\jmath}} R_{i \bar{\jmath} k \bar{l}}$ is the Ricci tensor at $z=0$ and $B_{k \bar{l}}=\delta^{i \bar{\jmath}} B_{i k \bar{\jmath}}$. We see that in this coordinate system the only solutions have $R_{k \bar{l}}=B_{k \bar{l}}=0$. The latter implies $B_{[i k][j]]}=0$ in $d \leq 3$.

\subsection{Higher Orders}

The appearance of Ricci flatness in the computation was somewhat unexpected, and to get more insight (and be sure that there are no further consistency conditions) we push the computation to the next non-trivial case, which is sixth order. In addition, we would like to know whether the uniqueness we found at fourth order persists at higher orders.

\subsection{Normal Coordinate Expansions to Sixth Order}

To this order, the expansion of the Kähler potential in normal coordinates is

$$
\begin{aligned}
& K_{0}(z, \bar{z})=z^{i} \bar{z}^{\bar{\imath}}+\sum_{p, q=2}^{\infty} K_{i_{1}, \ldots, i_{p} ; \bar{\jmath}_{1}, \ldots, \bar{j}_{q}}^{(p, q)} z^{i_{1}} \cdots z^{i_{p}} \bar{z}^{\bar{\jmath}_{1}} \cdots \bar{z}^{\bar{\jmath} q} \\
& =z^{i} \bar{z}^{i}-\frac{1}{4} R_{i \bar{\jmath} k l} z^{i} \bar{z}^{\bar{\jmath}} z^{k} \bar{z}^{\bar{l}} \\
& +S_{i \bar{\jmath} k \bar{m} m} z^{i} \bar{z}^{\bar{\jmath}} z^{k} \bar{z}^{\bar{l}} z^{m}+\text { c.c. } \\
& +T_{i \bar{\jmath} k \bar{l} m p} z^{i} \bar{z}^{\bar{\jmath}} z^{k} \bar{z}^{\bar{l}} z^{m} z^{p}+\text { c.c. } \\
& +U_{i \bar{\jmath} k \bar{l} m \bar{n}} z^{i} \bar{z}^{\bar{\jmath}} z^{k} \bar{z}^{\bar{l}} z^{m} \bar{z}^{\bar{n}} \\
& +\cdots,
\end{aligned}
$$

where the coefficients $S, T$ and $U$ are given by

$$
\begin{aligned}
S_{i \bar{\jmath} k \bar{l} m} & =-\left.\frac{1}{12} \frac{\partial}{\partial z^{m}} R_{i \bar{\jmath} k \bar{l}}(z)\right|_{z=0}, \\
T_{i \bar{\jmath} k \bar{l} m p} & =-\left.\frac{1}{48} \frac{\partial^{2}}{\partial z^{m} \partial z^{p}} R_{i \bar{i} \bar{l}}(z)\right|_{z=0}, \\
U_{i \bar{\jmath} k \bar{l} m \bar{n}} & =-\frac{1}{36}\left(\left.\frac{\partial^{2}}{\partial z^{m} \partial \bar{z}^{n}} R_{i \bar{\jmath} k l}(z)\right|_{z=0}-\delta^{p \bar{q}} R_{i \bar{q} k \bar{n}}(0) R_{p \bar{\jmath} m \bar{l}}(0)\right) .
\end{aligned}
$$

This can be checked by using the expression

$$
R_{i \bar{\jmath} k \bar{l}}(z)=-\frac{\partial^{4} K}{\partial z^{i} \partial \bar{z}^{\bar{\jmath}} \partial z^{k} \partial \bar{z}^{\bar{l}}}+g^{m \bar{n}} \frac{\partial^{3} K}{\partial z^{i} \partial z^{k} \partial \bar{z}^{\bar{n}}} \frac{\partial^{3} K}{\partial z^{m} \partial \bar{z}^{\jmath} \partial \bar{z}^{\bar{l}}} .
$$


The terms $S$ and $T$ correspond to purely holomorphic derivatives of the curvature and as such do not lead to essentially new features. However, the mixed derivatives $U z^{3} \bar{z}^{3}$ might.

Again by using (3.5) and (3.4), we find the expansion of the squared geodesic distance $d^{2}(x, z)$ to $O(z, \bar{z})^{6}$ :

$$
\begin{aligned}
& d^{2}(x, z)=|x-z|^{2} \\
& -\frac{1}{4} R_{i \bar{\jmath} k \bar{l}}(x-z)^{i}(\bar{x}-\bar{z})^{\bar{j}}(x+z)^{k}(\bar{x}+\bar{z})^{\bar{l}} \\
& -\frac{1}{12} R_{i \bar{\jmath} k l}(x-z)^{i}(\bar{x}-\bar{z})^{\bar{j}}(x-z)^{k}(\bar{x}-\bar{z})^{\bar{l}} \\
& +\frac{3}{4} S_{i \bar{\jmath} k \bar{l} m}(x-z)^{i}(\bar{x}-\bar{z})^{\bar{j}}(x+z)^{k}(\bar{x}+\bar{z})^{\bar{l}}(x+z)^{m} \\
& +\frac{1}{4} S_{i \bar{\jmath} k \bar{l} m}(x-z)^{i}(\bar{x}-\bar{z})^{\bar{\jmath}}(x-z)^{k}(\bar{x}+\bar{z})^{\bar{l}}(x-z)^{m} \\
& +\frac{1}{2} S_{i \bar{\jmath} k \bar{l}_{m}}(x-z)^{i}(\bar{x}-\bar{z})^{\bar{j}}(x-z)^{k}(\bar{x}-\bar{z})^{\bar{l}}(x+z)^{m} \\
& \text { +c.c. } \\
& +\frac{1}{10} T_{i \bar{\jmath} k \bar{l}_{m p}}(x-z)^{i}(\bar{x}-\bar{z})^{\bar{j}}(x-z)^{k}(\bar{x}-\bar{z})^{\bar{l}}(x-z)^{m}(x-z)^{p} \\
& +\frac{1}{2} T_{i \bar{\jmath} k \bar{l} m p}(x-z)^{i}(\bar{x}-\bar{z})^{\bar{\jmath}}(x+z)^{k}(\bar{x}-\bar{z})^{\bar{l}}(x+z)^{m}(x-z)^{p} \\
& +\frac{1}{2} T_{i \bar{\jmath} k \bar{l} m p}(x-z)^{i}(\bar{x}-\bar{z})^{\bar{j}}(x+z)^{k}(\bar{x}+\bar{z})^{\bar{l}}(x-z)^{m}(x-z)^{p} \\
& +\frac{1}{2} T_{i \bar{\jmath} k \bar{l}_{m p}}(x-z)^{i}(\bar{x}-\bar{z})^{\bar{j}}(x+z)^{k}(\bar{x}+\bar{z})^{\bar{l}}(x+z)^{m}(x+z)^{p} \\
& \text { +c.c. } \\
& +\frac{9}{80} U_{i \bar{\jmath} k \bar{l} \bar{n}}(x-z)^{i}(\bar{x}-\bar{z})^{\bar{j}}(x-z)^{k}(\bar{x}-\bar{z})^{\bar{l}}(x-z)^{m}(\bar{x}-\bar{z})^{\bar{n}} \\
& +\frac{3}{16} U_{i \bar{\jmath} k \bar{l}_{m}}(x-z)^{i}(\bar{x}-\bar{z})^{\bar{j}}(x+z)^{k}(\bar{x}-\bar{z})^{\bar{l}}(x+z)^{m}(\bar{x}-\bar{z})^{\bar{n}} \\
& +\frac{3}{4} U_{i \bar{\jmath} k \bar{l} m \bar{n}}(x-z)^{i}(\bar{x}-\bar{z})^{\bar{j}}(x+z)^{k}(\bar{x}+\bar{z})^{\bar{l}}(x-z)^{m}(\bar{x}-\bar{z})^{\bar{n}} \\
& +\frac{3}{16} U_{i \bar{\jmath} k \bar{l} \bar{n}}(x-z)^{i}(\bar{x}-\bar{z})^{\bar{j}}(x-z)^{k}(\bar{x}+\bar{z})^{\bar{l}}(x-z)^{m}(\bar{x}+\bar{z})^{\bar{n}} \\
& +\frac{9}{16} U_{i \bar{\jmath} k \bar{l}_{m} \bar{n}}(x-z)^{i}(\bar{x}-\bar{z})^{\bar{j}}(x+z)^{k}(\bar{x}+\bar{z})^{\bar{l}}(x+z)^{m}(\bar{x}+\bar{z})^{\bar{n}} \\
& -\frac{1}{720} R_{i \bar{q} k \bar{n}} \delta^{p \bar{q}} R_{p \bar{\jmath} m \bar{l}}(x-z)^{i}(\bar{x}-\bar{z})^{\bar{j}}(x-z)^{k}(\bar{x}-\bar{z})^{\bar{l}}(x-z)^{m}(\bar{x}-\bar{z})^{\bar{n}} \\
& -\frac{1}{48} R_{i \bar{q} k \bar{n}} \delta^{p \bar{q}} R_{p \bar{\jmath} m \bar{l}}(x-z)^{i}(\bar{x}-\bar{z})^{\bar{j}}(x-z)^{k}(\bar{x}-\bar{z})^{\bar{l}}(x+z)^{m}(\bar{x}+\bar{z})^{\bar{n}} \\
& +\cdots
\end{aligned}
$$

\subsection{D-Kähler Potential to Fifth Order}

We now consider the possible fifth order terms in the D-Kähler potential, and their contributions to $\hat{g}_{i \bar{j}}$. 
The natural guess for the fifth order term is

$$
K^{(5)}(Z, \bar{Z})=\ldots+S_{i \bar{\jmath} k \bar{l} m} \mathrm{STr} Z^{i} \bar{Z}^{\bar{j}} Z^{k} \bar{Z}^{\bar{l}} Z^{m}+\text { c.c. }+\ldots
$$

This leads to the variation

$$
\begin{aligned}
\hat{g}_{i \bar{\jmath}}^{(5)}(x, z)= & \frac{3}{4} S_{i \bar{\jmath} k \bar{l} m}(x+z)^{k}(\bar{x}+\bar{z})^{\bar{l}}(x+z)^{m} \\
& +\frac{1}{4} S_{i \bar{\jmath} k \bar{l} m}(x-z)^{k}(\bar{x}+\bar{z})^{\bar{l}}(x-z)^{m} \\
& +\frac{1}{2} S_{i \bar{\jmath} k \bar{l}_{m}}(x-z)^{k}(\bar{x}-\bar{z})^{\bar{l}}(x+z)^{m}+\text { c.c. },
\end{aligned}
$$

which indeed reproduces the corresponding terms of (5.11) in (5.2).

However, it is not the unique Kähler potential which does so. In fact, there are two commutator terms one can add which modify $\hat{g}_{i \bar{\jmath}}$, but which preserve both (5.2) and (5.3) at this order. An explicit example is

$$
\begin{aligned}
K^{(5)}(Z, \bar{Z})= & E_{[i j] k \bar{m} \bar{m}} \operatorname{Tr}\left[Z^{i}, Z^{j}\right]\left\{\left[Z^{k}, \bar{Z}^{\bar{l}}\right], \bar{Z}^{\bar{m}}\right\} \\
& +E_{[\bar{\imath}] \bar{l}] \bar{k} l m}^{*} \operatorname{Tr}\left[\bar{Z}^{\bar{\imath}}, \bar{Z}^{\bar{j}}\right]\left\{\left[\bar{Z}^{\bar{k}}, Z^{l}\right], Z^{m}\right\} .
\end{aligned}
$$

This leads to the variation

$$
\begin{aligned}
\hat{g}_{i \bar{\jmath}}^{(5)}(x, z)=2 E_{i l k \bar{\jmath} \bar{m}}(z-x)^{l}(x-z)^{k}(\bar{x}+\bar{z})^{\bar{m}} \\
\\
+2 E_{\bar{\jmath} \bar{k} i m}^{*}(\bar{z}-\bar{x})^{\bar{l}}(\bar{x}-\bar{z})^{\bar{k}}(x+z)^{m} .
\end{aligned}
$$

The symmetry of $E$ guarantees that this will reproduce (5.2), while at this order, the condition $\operatorname{det} \hat{g}=1$ reduces to $\operatorname{Tr} \hat{g}^{(5)}=0$, which also has solutions, e.g. $E_{i j k \bar{m} \bar{m}}=\epsilon_{i j} \delta_{k \bar{l}} c_{\bar{m}}$.

This means that the axioms as stated do not have a unique solution. To clarify the degree of non-uniqueness, let us check the Riemann curvature on the moduli space. One can show that the mixed components are given by

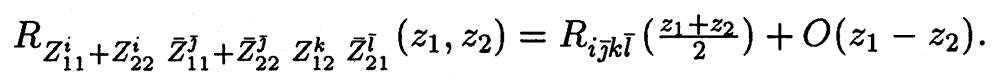

The ambiguities discussed above manifest themselves at $O\left(z_{1}-z_{2}\right)$. In terms of the geometry of the configuration space $\mathcal{X}_{N}$, the curvature on the bundle of off-diagonal modes is fixed on the diagonal $z_{i}=z_{j}$, but is not completely fixed off the diagonal.

\subsection{D-Kähler Potential to Sixth Order}

We give an explicit solution of the conditions to this order, without studying the question of uniqueness. 
The gauge boson mass condition (3.11) can be reproduced by the Kähler potential

$$
\begin{aligned}
& K(Z, \bar{Z})=\operatorname{Tr} Z \bar{Z} \\
& -\frac{1}{4} R_{i \bar{\jmath} k \bar{l}} \mathrm{~S} \operatorname{Tr} Z^{i} \bar{Z}^{\bar{\jmath}} Z^{k} \bar{Z}^{\bar{l}} \\
& +S_{i \bar{\jmath} k \overline{l m}} \mathrm{~S} \operatorname{Tr} Z^{i} \bar{Z}^{\bar{\jmath}} Z^{k} \bar{Z}^{\bar{l}} Z^{m}+\text { c.c. } \\
& +T_{i \bar{\jmath} k \overline{l m} p} \mathrm{~S} \operatorname{Tr} Z^{i} \bar{Z}^{\bar{j}} Z^{k} \bar{Z}^{\bar{l}} Z^{m} Z^{p}+\text { c.c. } \\
& +U_{i \bar{\jmath} k \bar{l} m \bar{n}} \mathrm{~S} \operatorname{Tr} Z^{i} \bar{Z}^{\bar{j}} Z^{k} \bar{Z}^{\bar{l}} Z^{m} \bar{Z}^{\bar{n}} \\
& +R_{i \bar{q} m \bar{n}} \delta^{\bar{q} p} R_{p \bar{l} k \bar{\jmath}} \\
& \times\left(-\frac{1}{96} \operatorname{Tr}\left\{Z^{k},\left[Z^{i}, \bar{Z}^{\bar{l}}\right]\right\}\left\{\bar{Z}^{\bar{n}},\left[Z^{m}, \bar{Z}^{j}\right]\right\}\right. \\
& \left.+\frac{1}{1440} \operatorname{Tr}\left[Z^{k},\left[Z^{i}, \bar{Z}^{\bar{l}}\right]\right]\left[\bar{Z}^{\bar{n}},\left[Z^{m}, \bar{Z}^{\jmath}\right]\right]\right) \\
& +F_{k i \bar{l} m[\bar{n} \bar{q}]} \operatorname{Tr}\left\{Z^{k},\left[Z^{i}, \bar{Z}^{\bar{l}}\right]\right\}\left\{Z^{m},\left[\bar{Z}^{\bar{n}}, \bar{Z}^{\bar{q}}\right]\right\}+\text { c.c. } \\
& +G_{k i \bar{l} m[\bar{n} \bar{q}]} \operatorname{Tr}\left[Z^{k},\left[Z^{i}, \bar{Z}^{\bar{l}}\right]\right]\left[Z^{m},\left[\bar{Z}^{\bar{n}}, \bar{Z}^{\bar{q}}\right]\right]+\text { c.c. } \\
& \begin{aligned}
= & \sum_{\substack{p, q \\
p+q \leq 6}} K_{i_{1}, \ldots, i_{p} ; \bar{\jmath}_{1}, \ldots, \bar{j}_{q}}^{(p, q)} \mathrm{STr} Z^{i_{1}} \cdots Z^{i_{p}} \bar{Z}^{\bar{\jmath}_{1}} \cdots \bar{Z}^{\overline{j q}} \\
& +R_{i \bar{q} m \bar{n}} \delta^{\bar{q} p} R_{p \bar{l} k \bar{\jmath}}
\end{aligned} \\
& \times\left(-\frac{1}{96} \operatorname{Tr}\left\{Z^{k},\left[Z^{i}, \bar{Z}^{\bar{l}}\right]\right\}\left\{\bar{Z}^{\bar{n}},\left[Z^{m}, \bar{Z}^{\jmath}\right]\right\}\right. \\
& \left.+\frac{1}{1440} \operatorname{Tr}\left[Z^{k},\left[Z^{i}, \bar{Z}^{\bar{l}}\right]\right]\left[\bar{Z}^{\bar{n}},\left[Z^{m}, \bar{Z}^{\bar{\jmath}}\right]\right]\right) \\
& +F_{k i \bar{l} m[\bar{\jmath} \bar{n}]} \operatorname{Tr}\left\{Z^{k},\left[Z^{i}, \bar{Z}^{\bar{l}}\right]\right\}\left\{Z^{m},\left[\bar{Z}^{\bar{j}}, \bar{Z}^{\bar{n}}\right]\right\}+\text { c.c. } \\
& +G_{k i \bar{l}_{m}[\bar{\jmath} n]} \operatorname{Tr}\left[Z^{k},\left[Z^{i}, \bar{Z}^{\bar{l}}\right]\right]\left[Z^{m},\left[\bar{Z}^{\bar{j}}, \bar{Z}^{\bar{n}}\right]\right]+\text { c.c. . }
\end{aligned}
$$

Note that it is not a symmetric trace. The extra $O\left(R^{2}\right)$ terms are required to satisfy (5.2), while the terms $F$ and $G$ will be required to satisfy (5.3).

This $K(Z, \bar{Z})$ gives $\hat{g}$ as

$$
\begin{aligned}
\hat{g}_{i \bar{\jmath}}(x, z):= & \left.\frac{\partial^{2}}{\partial Z_{12}^{i} \partial \bar{Z}_{21}^{j}} K\right|_{Z \in \mathcal{M}^{N} / S_{N}}= \\
= & \delta_{i \bar{\jmath}}-\frac{1}{4} R_{i \bar{\jmath} k \bar{l}}(x+z)^{k}(\bar{x}+\bar{z})^{\bar{l}}-\frac{1}{12} R_{i \bar{\jmath} k \bar{l}}(x-z)^{k}(\bar{x}-\bar{z})^{\bar{l}}= \\
& +\frac{1}{4} S_{i \bar{\jmath} k \bar{l} m}(x-z)^{k}(\bar{x}+\bar{z})^{\bar{l}}(x-z)^{m} \\
& +\frac{1}{2} S_{i \bar{\jmath} k \bar{l} m}(x-z)^{k}(\bar{x}-\bar{z})^{\bar{l}}(x+z)^{m}
\end{aligned}
$$




$$
\begin{aligned}
& +\frac{3}{4} S_{i j k \overline{l m}}(x+z)^{k}(\bar{x}+\bar{z})^{\bar{l}}(x+z)^{m}+\text { c.c. } \\
& +\frac{1}{10} T_{i \bar{\jmath} k \bar{l}_{m} p}(x-z)^{k}(\bar{x}-\bar{z})^{\bar{l}}(x-z)^{m}(x-z)^{p} \\
& +\frac{1}{2} T_{i \bar{\jmath} k \bar{l}_{m p}}(x+z)^{k}(\bar{x}+\bar{z})^{\bar{l}}(x-z)^{m}(x-z)^{p} \\
& +\frac{1}{2} T_{i \bar{\jmath} k \bar{l}_{m p}}(x-z)^{k}(\bar{x}-\bar{z})^{\bar{l}}(x+z)^{m}(x+z)^{p}
\end{aligned}
$$

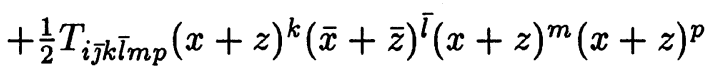

$$
\begin{aligned}
& \text { +c.c. } \\
& +\left(\frac{9}{80} U_{i \bar{\jmath} k \bar{l} m \bar{n}}-\frac{1}{720} R_{i \bar{q} k \bar{n}} \delta^{p \bar{q}} R_{p \bar{\jmath} m \bar{l}}+2 G_{k i \bar{l} m \bar{\jmath} \bar{n}}+\text { c.c. }\right) \\
& (x-z)^{k}(\bar{x}-\bar{z})^{\bar{l}}(x-z)^{m}(\bar{x}-\bar{z})^{\bar{n}} \\
& +\left(\frac{3}{4} U_{i \bar{\jmath} k \bar{l} m \bar{n}}-\frac{1}{48} R_{i \bar{q} k \bar{n}} \delta^{p \bar{q}} R_{p \bar{\jmath} m \bar{l}}\right)(x-z)^{k}(\bar{x}-\bar{z})^{\bar{l}}(x+z)^{m}(\bar{x}+\bar{z})^{\bar{n}} \\
& +\left(\frac{3}{16} U_{i \bar{\jmath} k \bar{l} m \bar{n}}+2 F_{k i \bar{l} m \bar{\jmath} \bar{n}}\right)(x-z)^{k}(\bar{x}+\bar{z})^{\bar{l}}(x-z)^{m}(\bar{x}+\bar{z})^{\bar{n}}+\text { c.c. } \\
& +\frac{9}{16} U_{i \bar{\jmath} k \bar{m} \bar{n}}(x+z)^{k}(\bar{x}+\bar{z})^{\bar{l}}(x+z)^{m}(\bar{x}+\bar{z})^{\bar{n}} \\
& +\cdots \text {. }
\end{aligned}
$$

\subsection{Ricci Flatness}

We are now in a position to check the consistency condition (5.3) at higher order.

On the one hand, we list the constraints on the coefficients $S T U$ following from Ricci flatness: given that

$$
\begin{aligned}
& 0 \equiv g^{i \bar{\jmath}}(z) R_{i \bar{\jmath} k \bar{l}}(z) \\
&=\left\{\delta^{i \bar{\jmath}}+\delta^{i \bar{q}} \delta^{\bar{\jmath} p} R_{p \bar{q} k l} z^{k} \bar{z}^{\bar{l}}+\cdots\right\} \times \\
&\left\{R_{i \bar{\jmath} k \bar{l}}-12\left(S_{i \bar{\jmath} k \bar{l} m} z^{m}+\text { c.c. }\right)-24\left(T_{i \bar{\jmath} k \bar{l} m p} z^{m} z^{p}+\text { c.c. }\right)\right. \\
&\left.\quad-36 U_{i \bar{\jmath} k \bar{l} \bar{n} \bar{n}} z^{m} \bar{z}^{\bar{n}}+\delta^{p \bar{q}} R_{i \bar{q} k \bar{n}} R_{p \bar{\jmath} m \bar{l}} z^{m} \bar{z}^{\bar{n}}+\cdots\right\},
\end{aligned}
$$

we have

$$
\begin{aligned}
& \delta^{i \bar{\jmath}} R_{i \bar{\jmath} k \bar{l}}=0 \\
& \delta^{i \bar{\jmath}} S_{i \bar{\jmath} k \bar{l} m}=0 \\
& \delta^{i \bar{\jmath}} T_{i \bar{\jmath} k \bar{l} m p}=0 \\
& \delta^{i \bar{\jmath}} U_{i \bar{\jmath} k \bar{l} m \bar{n}}=\frac{1}{36} \delta^{i \bar{\jmath}} \delta^{p \bar{q}}\left(R_{i \bar{q} k \bar{n}} R_{p \bar{\jmath} m \bar{l}}+R_{i \bar{q} k \bar{l}} R_{p \bar{\jmath} m \bar{n}}\right)
\end{aligned}
$$


for all $k, \bar{l}, m, \bar{n}, p$.

In order to compute det $\hat{g}$ to $O(z, \bar{z})^{4}$, let us put $\hat{g}_{i \bar{\jmath}}=\delta_{i \bar{\jmath}}+\hat{h}_{i \bar{\jmath}}$, and use the following formula

$$
\begin{aligned}
\operatorname{det} \hat{g} & =\operatorname{det}(1+\hat{h}) \\
& =\exp \operatorname{Tr} \log (1+\hat{h}) \\
& =\exp \left(\operatorname{Tr} \hat{h}-\frac{1}{2} \operatorname{Tr} \hat{h}^{2}+\cdots\right)
\end{aligned}
$$

(Note that $\hat{h}$ is $O\left(z^{1} \bar{z}^{1}\right)$, we can safely neglect higher powers of $\hat{h}$.) Thus, we need to check

$$
\operatorname{Tr} \hat{h}-\frac{1}{2} \operatorname{Tr} \hat{h}^{2}=O(z, \bar{z})^{5} .
$$

Plugging $\hat{h}$ from (5.18) into (5.22), we immediately get

$$
\begin{array}{ll}
O\left(z^{1} \bar{z}^{1}\right) \Rightarrow & \delta^{i \bar{\jmath}} R_{i \bar{\jmath} k l}=0 \\
O\left(z^{2} \bar{z}^{1}\right) \Rightarrow & \delta^{i \bar{\jmath}} S_{i \bar{\jmath} k \bar{l} m}=0 \\
O\left(z^{3} \bar{z}^{1}\right) \Rightarrow & \delta^{i \bar{\jmath}} T_{i \bar{\jmath} k \bar{l} m p}=0 .
\end{array}
$$

from lower order terms as a necessary condition for $\operatorname{det} \hat{g} \equiv 1$. Clearly, these follow from Ricci flatness (5.20).

This is not the case for $O\left(z^{2} \bar{z}^{2}\right)$ terms. More precisely, the coefficients of the following terms cancel using the last equation of (5.20):

$$
\begin{aligned}
& (x+z)^{k}(\bar{x}+\bar{z})^{\bar{l}}(x+z)^{m}(\bar{x}+\bar{z})^{\bar{n}}, \\
& (x-z)^{k}(\bar{x}-\bar{z})^{\bar{l}}(x+z)^{m}(\bar{x}+\bar{z})^{\bar{n}} .
\end{aligned}
$$

Those associated with

$$
\begin{aligned}
& (x-z)^{k}(\bar{x}-\bar{z})^{\bar{l}}(x-z)^{m}(\bar{x}-\bar{z})^{\bar{n}}, \\
& (x-z)^{k}(\bar{x}+\bar{z})^{\bar{l}}(x-z)^{m}(\bar{x}+\bar{z})^{\bar{n}} \\
& (x+z)^{k}(\bar{x}-\bar{z})^{\bar{l}}(x+z)^{m}(\bar{x}-\bar{z})^{\bar{n}}
\end{aligned}
$$

do not automatically cancel, but can be made to cancel by appropriate choice of the terms $F$ and $G$ :

$$
\begin{aligned}
& F_{k i \bar{l} m[\bar{\jmath} \bar{n}]}=+\frac{1}{192}\left(R_{i \bar{q} m \bar{n}} \delta^{\bar{q} p} R_{p \bar{l} k \bar{j}}-R_{i \bar{q} m \bar{\jmath}} \delta^{\bar{q} p} R_{p \bar{l} \bar{k} \bar{n}}\right) \text {, } \\
& G_{k i \bar{l} m[\bar{\jmath} \bar{n}]}=-\frac{1}{2880}\left(R_{i \bar{q} m \bar{n}} \delta^{\bar{q} p} R_{p \bar{p} k \bar{j}}-R_{i \bar{q} m \bar{\jmath}} \delta^{\bar{q} p} R_{p \bar{l} k \bar{n}}\right) \text {. }
\end{aligned}
$$

In conclusion, one can find a sixth order $K(Z, \bar{Z})$ such that both the gauge boson mass condition and det $\hat{g} \equiv 1$ are satisfied. 


\section{Conclusions}

In this work we found actions for $N \mathrm{D} p$-branes sitting at points in a threecomplex dimensional Kähler manifold, satisfying natural conditions from D-brane physics, notably the enhancement of gauge symmetry when the Dbranes coincide, and the proportionality of the mass of a string stretched between two D-branes to the shortest geodesic distance between them.

These actions would be expected to arise as the low energy limit of Dbrane actions derived from string theory for manifolds with weak curvature. They are also natural starting points for the definition of $M$ (atrix)theory $[7,8]$ on target space $\mathbb{R}^{5-p} \times T^{p} \times \mathcal{M}$, where $\mathcal{M}$ is topologically trivial but curved, or a subregion of a larger compact manifold. The condition on the string masses guarantees that the one-loop quantum effective action will contain a term $v^{4} / d^{7-p}$, the leading short distance behavior of the supergravity interaction in this case [9]. Now this is not to say that the action as we have computed it so far is a complete and consistent definition of M(atrix)theory in this background - it seems likely that additional terms higher order in commutators as well as in derivatives would be required to get the physics right - but rather that the consistency conditions which we can check at this order can be satisfied and with a pleasing degree of uniqueness.

We found that the mass condition cannot be satisfied unless the manifold is Ricci flat, or (combining with the Kähler condition) a Calabi-Yau manifold. Now at first this might not seem surprising - certainly we need to start with a consistent closed string theory background to define sensible open string actions. However, the approach taken here only used rather general consistency conditions, and no details of string theory. The standard string theory argument, world-sheet conformal invariance expressed as the RG fixed point condition on the world-sheet sigma model, does not have any obvious connection with the starting point or the analysis.

Furthermore, the claim that these actions are appropriate starting points for $M$ (atrix)-theory certainly suggests that we should look for an argument independent of string theory. What we are saying in this context is that two a priori independent consistency conditions on the physics - that the background satisfy the equations of motion, and that the one-loop quantum corrections reproduce supergravity interactions - are in fact related.

Another known argument for Ricci flatness of target spaces for brane theories which may have a closer relation to the present story is the requirement for kappa symmetry of the covariant supermembrane action that the background satisfy the supergravity equation of motion $[10,11]$. Now, according to the rules of $\mathrm{M}$ (atrix)-theory, we can find membrane solutions of 
the action, leading to a possible relation; on the other hand we are necessarily working in light-cone gauge, where kappa symmetry has already been fixed.

A related argument which could work after gauge fixing is due to Aharony, Kachru and Silverstein (unpublished). String theory or M-Theory compactified on a Calabi-Yau target will have $N=2, d=4$ (or the equivalent $N=1, d=5$ ) supersymmetry, and the branes will break half of this. On the other hand, if the space is not Ricci flat, one would argue that since there is no covariantly constant spinor, there is no unbroken supersymmetry, and the D-brane theory cannot be supersymmetric. Thus, given that we assumed that the D-brane theory is supersymmetric, we should find that the target space has a covariantly constant spinor and is thus Calabi-Yau.

Although this is an attractive argument, the problem with it (recognized by AKS as well) is that the true condition for supersymmetry in string theory is that the target space have zero integrated Ricci curvature (zero first Chern class). We expect $\alpha^{\prime}$ corrections to the target space metric, and these can be compatible with supersymmetry, if we also have $\alpha^{\prime}$ corrections to the supersymmetry transformation laws, which modify the condition for an unbroken supersymmetry away from Ricci flatness.

Our result adds to this the statement that the exact proportionality of the masises of stretched strings to the geodesic distance must also gain $\alpha^{\prime}$ corrections in this case; we know no argument for or against this in general. For example, the $\mathbb{C}^{3} / \Gamma$ orbifold models in [12], which generically describe D-brane propagation on non-Ricci flat metrics, will not satisfy the isotropic mass condition in these cases. On the other hand, if one can tune the "seed" metric in that construction to produce a Ricci flat physical metric, it will be interesting to go on and implement the mass condition.

It will be interesting to recast our discussion in more geometrical language, and find axioms which further constrain the action and determine the higher commutator terms. The way in which the Ricci-flatness condition arose in our considerations, through the equation (5.3), suggests that the full configuration space $\mathcal{X}_{N}$ must be Calabi-Yau.

A very interesting question is whether the mass condition is stable under quanturn corrections. Since these are non-renormalizable sigma models, the question seems best defined for $p \leq 1$. For $p=1$, it would appear to be true at one loop, if the full configuration space is indeed Calabi-Yau. On the other hand, one might worry that the known four-loop beta function would violate it. Perhaps the coupling to the gauge fields changes this? For $p=0$, we do not have renormalization in the conventional sense, but it seems quite possible that some problems involving large $N$ numbers of $\mathrm{D}$-branes can be treated by a large $N$ renormalization group. Some comments on this are 
made in [3]; for both types of renormalization, it will be very interesting to look at quantum corrections in these models.

\section{Acknowledgements}

We thank B. de Wit, D.-E. Diaconescu and M. Kontsevich for useful conversations. M.R.D. thanks Cern for hospitality. A.K. would like to thank the Theory Group of Lawrence Berkeley National Laboratory for hospitality. H.O. thanks Physics Departments of Rutgers, Harvard Universities, Laboratoire de Physique Théorique et Hautes Energies (L.P.T.H.E.) at Universités Pierre et Marie Curie (Paris VI), and Aspen Center for Physics for hospitality.

M.R.D. is supported in part by DOE grant DE-FG05-90ER40559. H.O. is supported in part by NSF grant PHY-95-14797 and DOE grant DE-AC0376SF00098. A.K is supported by the fellowship from the Japanese Ministry of Education, Science and Culture.

\section{References}

[1] J. Dai, R. G. Leigh and J. Polchinski, Mod. Phys. Lett. A4 (1989) 2073; J. Polchinski, Phys. Rev. Lett. 75 (1995) 4724.

[2] R. Leigh, Mod. Phys. Lett. A4 (1989) 2767.

[3] M. R. Douglas, "D-branes and M(atrix)-theory in Curved Space", lecture at Strings '97, hep-th/9707228.

[4] M. R. Douglas, Adv. Theor. Math. Phys. 1 (1997) 198.

[5] A. Sen, Phys. Rev. D55 (1997) 2501.

[6] J. Hadamard, Lectures on Cauchy's Problem in Linear Partial Differential Equations, Yale University Press 1923 and Dover 1952; as discussed in J. J. Duistermaat, The Heat Kernel Lefschetz Fixed Point Formula for the Spin-C Dirac Operator, Birkhäuser 1996.

[7] T. Banks, W. Fischler, S. H. Shenker and L. Susskind, Phys. Rev. D55 (1997) 5112.

[8] T. Banks, "The State of M(atrix)-theory", hep-th/9706168.

[9] M. R. Douglas, H. Ooguri and S. H. Shenker, Phys. Lett. B402 (1997) 36. 
[10] E. Bergshoeff, E. Sezgin, P.K. Townsend, Phys. Lett. B189 (1987) 75; Ann. Phys. 185 (1988) 330.

[11] M.J. Duff, P.S. Howe, T. Inami, K.S. Stelle, Phys. Lett. B191 (1987) 70 .

[12] M. R. Douglas and B. R. Greene, Adv. Theor. Math. Phys. 1 (1997) 184. 